\title{
GMR
}

\section{A genome-wide association study of growth trait-related single nucleotide polymorphisms in Chinese Yancheng chickens}

\author{
C.F. Jin, Y.J. Chen, Z.Q. Yang, K. Shi and C.K. Chen \\ Institute of Agricultural Sciences of Yanchen and Coastal Area of Jiangsu Province, \\ Yancheng, China \\ Corresponding author: Y.J. Chen \\ E-mail: ycaasjin@163.com
}

Genet. Mol. Res. 14 (4): 15783-15792 (2015)

Received August 19, 2015

Accepted October 23, 2015

Published December 1, 2015

DOI http://dx.doi.org/10.4238/2015.December.1.30

ABSTRACT. Chicken (Gallus gallus) growth traits are important economic traits, and many studies have been conducted on genetic selection for body weight. However, most of these studies have detected functional chromosome mutations or regions by conventional molecular markers or gene chips. In this study, we performed a new genome-wide association study using specific-locus amplified fragment sequencing (SLAFseq) technology in purebred Yancheng chickens. Single nucleotide polymorphisms (SNPs) that were significantly associated with phenotypic traits were identified by GAPIT-compressed mixed linear models. Eighteen SNP markers reached $5 \%$ Bonferroni genome-wide significance. A region spanning 72.3 to $82.1 \mathrm{Mb}$ on GGA4 had a strong influence on growth traits. Four genes (FAM184B, KCNIP4, MIR15A, and GLI3) were closely associated with body weight. Some SNPs were coincident with previously reported quantitative trait locus regions. Our results would promote the researches of Chinese chickens and accelerate the utilization of Chinese chicken, specially Yancheng chicken.

Key words: Yancheng chicken; SLAF-seq; Genome-wide association study; GWAS 


\section{INTRODUCTION}

Growth traits are among the most important economic traits in the poultry industry. Remarkable advances in the study of growth traits have been achieved, and many relative genes and quantitative trait loci (QTLs) have been discovered (Sewalem et al., 2002; Carlborg et al., 2004; Jacobsson et al., 2005; Tsudzuki et al., 2007; Moura et al., 2009; Wahlberg et al., 2009). More than 1500 QTLs, covering most of the chicken genome, are associated with growth traits ( $\mathrm{Hu}$ et al., 2010). Previous genomic studies have generally employed low-density microsatellites as markers; however, this approach can no longer provide novel information. Recently, the genomewide association study (GWAS) technique was developed and used to search for single nucleotide polymorphisms (SNPs) and functional genes that affect quantitative traits. A GWAS does not assume that certain genes or QTLs are associated with specific traits (Hardy and Singleton, 2009), but is rather a genome-wide study of the association between given traits and genetic markers (Liu et al., 2008; McCarthy et al., 2008; Cho et al., 2009). Most previous studies have used gene chips, but a new and efficient solution for large-scale genotyping called specific-locus amplified fragment sequencing (SLAF-seq) has been developed. SLAF-seq technology has several distinguishing characteristics: i) deep sequencing to ensure genotyping accuracy; ii) reduced representation strategy to reduce sequencing costs; iii) a pre-designed reduced representation scheme to optimize marker efficiency; and iv) a double-barcode system for large populations (Sun et al., 2013). In the present study, a GWAS was conducted using SLAF-seq technology in order to identify SNPs that are significantly associated with the growth traits of a Chinese chicken breed.

\section{MATERIAL AND METHODS}

\section{Ethics statement}

All of the experimental procedures were conducted in conformity with institutional guidelines for the care and use of laboratory animals, and the protocols were approved by the Institutional Animal Care and Use Committee of the Ethics Institute, Jiangsu Academy of Agricultural Sciences, China.

\section{Experimental animals}

The animals used in this study were obtained from the Jinghai Yellow Chicken Breeding Station. The Yancheng chicken is a combination of broiler and layer chickens. It has a thin skin and tender, tasty meat. Yancheng chickens are responsive, active, and lively, and their color is yellow; they exhibit coarse-feeding resistance, disease resistance, social and field foraging ability, high performance, and are suitable for coastal climates and conditions. Two hundred purebred hens of the same batch from the same generation were randomly selected. All of the chickens had complete genealogical records and were reared in stair-step caging under the same recommended nutritional and environmental conditions. The body weights of the 200 chickens were measured biweekly from birth to 16 weeks of age. The statistics of nine growth measurements are presented in Table 1.

\section{Genotyping}

DNA was extracted from blood samples using the phenol/chloroform method and diluted to $100 \mu \mathrm{g} / \mu \mathrm{L}$. Genomic DNA concentration and quality were assayed using a NanoDrop ${ }^{\mathrm{TM}} 2000$ 
spectrophotometer to ensure that they met genotyping requirements. SLAF-seq is an efficient method of large-scale genotyping, which is based on a reduced representation library and highthroughput sequencing. The procedure is shown in Figure 1. The Gallus gallus sequences were analyzed using SLAF_Predict (Biomarker, Beijing, China), based on the GC content, repeat sequences, and gene characteristics. The plan for marker development was designed by defining the enzyme digestion scheme, the gel cutting ranges, and the sequencing quantity, which were used to verify the density and homogeneity of the markers under development and ensure the successful preparation of the expected target.

\begin{tabular}{|c|c|c|c|c|}
\hline Measurement & Mean & Standard error & Minimum & Maximum \\
\hline $\mathrm{BW}^{1}$ & 32.5 & 0.58 & 20 & 45 \\
\hline BW2 & 95.4 & 1.28 & 73 & 136 \\
\hline BW4 & 192.5 & 4.90 & 145 & 302 \\
\hline BW6 & 378.7 & 41.30 & 260 & 520 \\
\hline BW8 & 479.9 & 11.50 & 355 & 675 \\
\hline BW10 & 689.4 & 45.40 & 550 & 862 \\
\hline BW12 & 859.3 & 27.60 & 640 & 1060 \\
\hline BW14 & 979.7 & 45.50 & 795 & 1355 \\
\hline BW16 & 1106.9 & 23.40 & 970 & 1520 \\
\hline
\end{tabular}

${ }^{1}$ The unit of body weight (BW) is the gram. 0, 2, 4, etc., refer to the week in which the BW measurement was taken.

An SLAF library was constructed in accordance with the pre-designed scheme. Haelll (New England Biolabs) was used to digest the genomic DNA. A single nucleotide A overhang was then added to the digested fragments using the Klenow fragment ( $3^{\prime} \rightarrow 5^{\prime}$ exo-) (New England Biolabs) and dATP at $37^{\circ} \mathrm{C}$. Duplex Tag-labeled sequencing adapters [polyacrylamide gel electrophoresis (PAGE) purified, Life Technologies] were then ligated to the A-tailed fragments using T4 DNA ligase. A polymerase chain reaction (PCR) was performed using diluted restriction-ligation DNA samples, dNTP, Q5 ${ }^{\circledR}$ High-Fidelity DNA Polymerase, and the PCR primers 5'-AATGATACGGCGACCACCGA-3' and 5'-CAAGCAGAAGACGGCATACG-3' (PAGE purified). The PCR products were purified using Agencourt AMPure XP beads (Beckman Coulter, High Wycombe, UK) and then pooled. The pooled sample was separated by electrophoresis on a $2 \%$ agarose gel. Fragments that were between 500 and 800 bp in size (with indexes and adaptors) were excised and purified using a QIAquick Gel Extraction Kit (Qiagen). The gel-purified products were sequenced on an Illumina HiSeq 2500 system (Illumina Inc., San Diego, CA, USA) according to the manufacturer protocol.

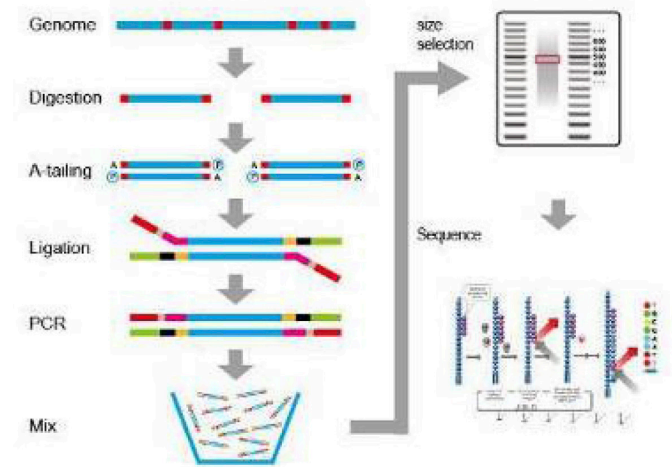

Figure 1. Flowchart of the specific-locus amplified fragment sequencing (SLAF-seq) method. 
Raw reads were sorted to individuals according to the barcode sequences, and lowquality reads (quality score $<20$ ) were filtered out. We evaluated and mapped the eligible reads using the SOAP 2.20 software (Li et al., 2009) to assemble newly referenced genomes (http://

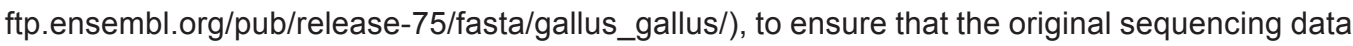
were effectively obtained. We deemed that the reads with double ends were reliable compared to the only locus of the genome, and could do SLAF label development. We chose the group whose average depth of sequencing was not less than 4 to define the SLAF label, in line with the comparison error correction result. PLINK (1.07) (Purcell et al., 2007) was used to perform data quality control. SNPs with a low call frequency $(<85 \%)$ and a low minor allele frequency $(<5 \%)$ were rejected.

\section{Statistical analysis}

We used the ADMIXTURE 1.22 software (Alexander et al., 2009) to calculate the samples' group structure, based on the SNPs. We assumed that the 200 samples' subgroup number $(Q$ value) was 1-10 for the cluster analysis, and confirmed the number of subgroups by their peak $\Delta Q$ value positions. The subgroups with a minimum $\triangle Q$ peak value were deemed the best.

The SNPs that were significantly associated with phenotypic traits were identified using a GAPIT compressed mixed linear model (MLM, II), which was as follows:

$$
y=X \alpha+Q \beta+K \mu+e
$$

where $Y$ is the phenotypic value, $X$ is the genotype, $Q$ is the population structure matrix calculated by the ADMIXTURE program, $\beta$ is the weight vector of each group, $K$ is the relative kinship matrix, $\alpha$ is the weight vector of each marker, and $e$ is the random error. $K$ was constructed from all of the SNPs using the SPAGeDi 1.3a software (Hardy and Vekemans, 2002). The threshold P value of the $5 \%$ Bonferroni genome-wide significance was based on the estimated number of independent markers and linkage disequilibrium (LD) blocks for autosome markers (Nicodemus et al., 2005). We assessed the population structure using ADMIXTURE, and all of the autosomal SNPs were pruned using the indep-pairwise option, with a window size of 25 SNPs, a step of five SNPs, and an $r^{2}$ threshold of 0.2 (Wang et al., 2009). An LD block was defined as a set of contiguous SNPs that had pairwise $r^{2}$ values exceeding 0.4 . We estimated the number of independent SNP markers using this approach. Given that the different genetic backgrounds of the population might lead to population stratification, we evaluated the population stratification status using ADMIXTURE. A quantile-quantile (Q-Q) plot was drawn to detect the population stratification for each trait.

\section{RESULTS}

\section{SLAF-seq results}

The distribution of all of the SLAFs in the genomes of the 200 samples was determined by the number of SLAFs per $100 \mathrm{~kb}$ in the genomes (Figure 2). SLAFs were relatively evenly distributed throughout the genomes, which indicated that the SLAF data were reliable. We then detected the SNPs among the defined SLAF fragments. The SLAF marker information for each chromosome is summarized in Table 2. 


$$
\frac{\Gamma}{\square I}
$$




\section{Group structure and cluster analysis}

The result of calculating the samples' group structure indicated that a $Q$ value of 1 was the lowest peak value (Figure 3), meaning that samples could be classified into one group. Regarding the Bonferroni $\mathrm{P}$ value, the total number of independent markers and LD blocks was 28,976 . Therefore, at a threshold $P$ value of $5 \%$, the Bonferroni genome-wide significance was 1.72E-06 $(0.05 / 28,976)$. Inter-individual genetic relationships may have influenced the population stratification; therefore, $Q-Q$ plots of body weight at eight time points were drawn (Figure 4). The observed values (ordinates) were calculated by association analysis and fitted to the expected values (abscissae), which indicated that there was no population stratification. Therefore, the association analysis result was reliable using the GAPIT mixed linear model.
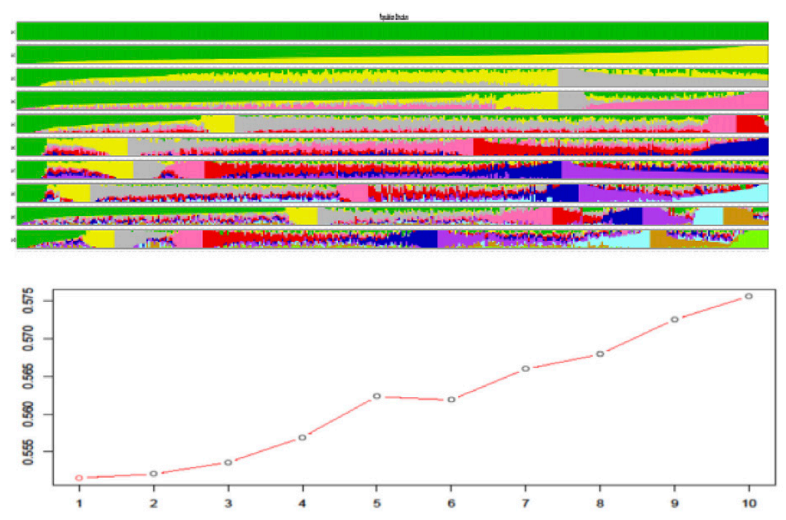

Figure 3. Group structure clustering. In the upper panel, each color represents a group and each line represents a cluster. The lower panel displays the clustering, where the cluster number ranges from 1 to 10 .

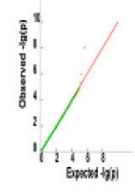

BW0

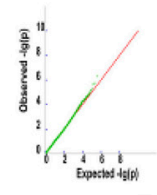

BW2

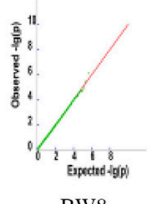

BW8

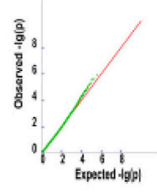

BW14

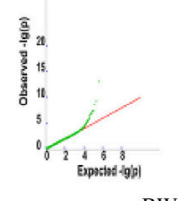

BW4
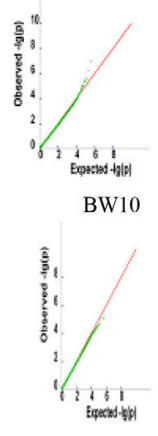

BW16

Figure 4. Quantile-quantile plots of body weight at eight time points. The ordinates are higher than the abscissae, which indicated that there is no population stratification. 


\section{Genome-wide association analysis}

According to the quality control criteria, 200 chickens and 89,560 SNPs that were distributed on 29 autosomes and the Z chromosome were eligible for genome-wide analysis. Based on the GAPIT mixed linear model and a Bonferroni correction, 18 SNPs exhibited genomewide associations with growth traits in Yancheng chickens $(P<1.72 E-06)$. We searched for these SNPs in the National Center for Biotechnology Information dbSNP database (http://www.ncbi.nlm. nih.gov/snp/) according to their position in the genome, in order to obtain their SNP ID and nearest genes (Table 3).

\begin{tabular}{|c|c|c|c|c|c|c|}
\hline Trait & SNP ID & Chr & Pos. (bp) ${ }^{1}$ & MAF & $P$ value & Nearest gene \\
\hline BW0 & rs13840709 & 1 & $25,098,872$ & 0.375 & 4.25E-08 & Novel gene \\
\hline BW2 & rs15990496 & 2 & $51,360,125$ & 0.3548 & 8.71E-07 & GLI3 \\
\hline BW2 & rs15229556 & 26 & 1,958,988 & 0.4785 & 1.71E-06 & RBBP5 \\
\hline BW4 & rs 16444875 & 4 & $82,169,307$ & 0.375 & $4.54 \mathrm{E}-14$ & CFAP99 \\
\hline BW4 & rs15368284 & 1 & $103,569,548$ & 0.3548 & 7.98E-09 & GRIK1 \\
\hline BW4 & rs 16023603 & 4 & $75,511,560$ & 0.2247 & $6.91 \mathrm{E}-08$ & FAM184B \\
\hline BW4 & rs15990496 & 2 & $51,360,125$ & 0.4126 & $8.71 \mathrm{E}-07$ & GLI3 \\
\hline BW4 & rs13701151 & 3 & $96,483,790$ & 0.2967 & 2.60E-07 & ATP6V1C2 \\
\hline BW4 & rs 14144201 & 2 & $16,380,387$ & 0.2063 & 4.69E-07 & GPR158 \\
\hline BW6 & rs 16023603 & 4 & $75,511,560$ & 0.2071 & 2.14E-07 & FAM184B \\
\hline BW6 & rs15498187 & 1 & $168,713,010$ & 0.4329 & $3.71 \mathrm{E}-07$ & $8 D$ MIR $15 A$ \\
\hline BW10 & GGaluGA265806 & 4 & $74,347,058$ & 0.3548 & 1.16E-07 & KCNIP4 \\
\hline BW10 & rs 16023603 & 4 & $75,511,560$ & 0.375 & 1.36E-06 & FAM184B \\
\hline BW10 & rs 15498187 & 1 & $168,713,010$ & 0.2336 & 1.63E-06 & $8 D$ MIR $15 A$ \\
\hline BW12 & rs14085822 & 3 & $11,691,800$ & 0.2987 & 4.44E-07 & CDC42BPA \\
\hline BW12 & rs16023603 & 4 & $75,511,560$ & 0.1899 & $1.59 \mathrm{E}-06$ & FAM184B \\
\hline BW14 & GGaluGA265806 & 4 & $74,347,058$ & 0.0404 & $1.25 \mathrm{E}-06$ & KCNIP4 \\
\hline BW14 & rs15938574 & 2 & $31,538,341$ & 0.375 & 1.39E-06 & 20 D STK31 \\
\hline
\end{tabular}

${ }^{1}$ SNP positions are based on WADHUC2 and SNP ID codes from the dbSNP database. MAF = minor allele frequency.

\section{DISCUSSION}

SLAF-seq technology was developed based on high-throughput sequencing. SLAF-seq is more appropriate for GWAS than using SNP chips, can obtain millions of SNPs at once, and detect novel SNPs on the genome (Xu et al., 2015). SLAF-seq can be applied to species with a reference genome, as well as to species without a reference genome (Sun et al., 2013).

SNPs reaching $5 \%$ genome-wide significance in this study were located on four different chromosomes. An obvious central region of GGA4 (72.3-82.1 Mb) was identified. This result agrees with the results of Gu et al. (2011), who used an F2 resource population derived from Silky Fowl and White Plymouth Rock chickens to detect SNPs associated with body weight at 7-14 weeks, and identified a region of GGA4 (71.6-80.2 Mb). Zhang et al. (2012) performed a GWAS with Beijing fatty chicken, and found no associated SNPs in the central region. Xie et al. (2012) identified SNPs in a region of GGA1 (173.5-175 Mb) that strongly affected the body weights of F2 chickens, which were based on a cross of Xinghua and White Plymouth Rock chickens. Differences between these results may have been caused by breed differences.

Eighteen SNPs and 12 valuable genes were detected in this study. Among the SNPs in these genes, the largest effects were exerted by rs15990496 in GLI3 for BW2 and BW4; rs 16023603 in FAM184B for BW4, BW6, BW10, and BW12; GGaluGA265806 in KCNIP4 for BW10 and BW14; 
and rs15498187 in 8DMIR15A for BW6 and BW10. FAM184B (family with sequence similarity, member B) can influence cattle ingestion, average daily gain, and carcass weight (LindholmPerry et al., 2011). In addition, a gene called NCAPG (non-SMC condensin I complex, subunit $\mathrm{G})$ that is $1 \mathrm{~kb}$ downstream of $F A M 184 b$ encodes a subunit of condensation protein complexes. NCAPG is a new candidate gene for cattle growth and carcass traits, and the polymorphism of this gene can influence the growth, bodily form, and carcass traits of different varieties of cattle at different times (Eberlein et al., 2009). Although FAM184B has not been reported in chickens, it could be a new candidate gene that influences chicken growth traits because of its important biological function. KCNIP4 belongs to a family of potassium channel-interacting proteins, and is highly expressed in normal kidney cells (Bonne et al., 2007). Potassium channels have extensive physiological adjustment functions, including neurotransmitter release, contraction of smooth muscle, heart rate adjustment, and insulin secretion. Therefore, we hypothesize that KCNIP4 has a significant effect on chicken growth. Regarding GL/3 and 8DMIR15A, a previous study found that these genes are important in controlling brain development (Laclef, 2014), which partly demonstrates that this gene affects growth traits. 8DMIR15A encodes a type of noncoding regulatory RNA, and is expressed in several chicken tissues, particularly the lung. 8DMIR15A influences many kinds of oncogenes, such as BCL2, MCL1, CCND1, and WNT3A (Galin et al., 2008; Aqeilan et al., 2010).

Regarding the other genes found in this study, STK31 is a member of the TDRD protein family. In mice, this protein has a significant effect on the development of middle-late germ cells and fertilization (Bao et al., 2012), and may have a similar function in chickens. GPIK1 is the main neurotransmitter receptor in mammals, and participates in a variety of neural physiological processes. This gene has also been identified as an important candidate anti-epileptic gene (Mulle et al., 1998). The CDS42BPA protein specifically binds CDC42, a phosphoglycerate kinase enzyme that regulates morphological changes in the cell, cell migration, the swallowing function, and the cell-cycle progression (Gong et al., 1997). Therefore, CDC42BPA may affect chicken growth via CDC42. GPR158 can control G-protein signaling pathways by regulating the position of the RGS7 complex, and its family member GPR120 is involved in human fat metabolism (Watson et al., 2012). Two other genes play important roles in biological processes: in Piétrain pigs, ATP6V1C2 functions in the negative regulation of apoptosis, immune responses, cell-cell signaling, cell growth and migration, and metabolic processes (Ropka-Molik et al., 2014); RBBP5 controls the efficiency of FOS transcript processing (Teoh and Sharrocks, 2014). We could not find any reports concerning the function of the CFAP999 gene product.

In summary, we identified 18 SNPs with genome-wide significance. Several SNPs were associated with more than one body weight trait. Most of the SNPs have not previously been reported in chickens, although some overlapped with QTLs for growth traits that have been described in previous studies. Twelve candidate genes that affect growth traits were also detected in our study, and according to previous reports, nearly all of these genes have important biological functions that might regulate the growth of Yancheng chickens. These candidate genes require further study. These results will benefit those involved in the research and utilization of Yancheng chickens, as well as other Chinese chicken breeds.

\section{Conflicts of interest}

The authors declare no conflict of interest. 


\section{ACKNOWLEDGMENTS}

Research supported by Projects in Agricultural Science and Technology Innovation of Jiangsu Province [\#CX(14)5091].

\section{REFERENCES}

Alexander DH, Novembre J and Lange K (2009). Fast model-based estimation of ancestry in unrelated individuals. Genome Res. 19: 1655-64. Doi: 10.1101/gr.094052.109.

Aqeilan R, Calin G and Groce C (2010). miR-15a and miR-16-1 in cancer: discovery, function and future perspectives. Cell Death 17: 215-220.

Bao J, Yuan S, Maestas A, Bhetwal BP, et al. (2012). Stk31 is dispensable for embryonic development and spermatogenesis in mice. Mol. Reprod. Dev. 80: 786-787.

Bonne A, Vreede L, Kuiper RP, Bodmer D, et al. (2007). Mapping of constitutional translocation breakpoints in renal cell cancer patients: identification of KCNIP4 as a candidate gene. Cancer Genet. Cytogenet. 179: 11-18.

Carlborg O, Hocking PM, Burt DW and Haley CS (2004). Simultaneous mapping of epistatic QTL in chickens reveals clusters of QTL pairs with similar genetic effects on growth. Genet. Res. 83: 197-209.

Cho YS, Go MJ, Kim YJ, Heo JY, et al. (2009). A large-scale genome-wide association study of Asian populations uncovers genetic factors influencing eight quantitative traits. Nat. Genet. 41: 527-534.

Eberlein A, Takasuga A, Setoguchi K, Pfuhl R, et al. (2009). Dissection of genetic factors modulating fetal growth in cattle indicates a substantial role of the non-SMC condensin I complex, subunit G (NCAPG) gene. Genetics 183: 951-964.

Galin GA, Cimmino A, Fabbri M, Ferracin M, et al. (2008). MIR-15a and miR-16-1 cluster functions in human leukemia. Proc. Natl. Acad. Sci. U. S. A. 105: 5166-5171.

Gong T-W, Shin JJ, Burmeister M and Lomax MI (1997). Complete cDNAs for CDC42 from chicken cochlea and mouse liver. Biochim. Biophys. Acta 1352: 282-292.

Gu X, Feng C, Ma L, Song C, et al. (2011). Genome-wide association study of body weight in chicken F2 resource population. PLoS One 6: e21872.

Hardy J and Singleton A (2009). Genomewide association studies and human disease. N. Eng. J. Med. 360: 1759-1768.

Hardy OJ and Vekemans X (2002). SPAGeDi: a versatile computer program to analyse spatial genetic structure at the individual or population levels. Mol. Ecol. Notes 2: 618-620.

Hu Z, Park C, Fritz E and Reecy J (2010). QTLdb: a comprehensive database tool building bridges between genotypes and phenotypes. The 9th World Congress on Genetics Applied to Livestock Production, Leipzig, 1-6.

Jacobsson L, Park HB, Wahlberg P, Fredriksson R, et al. (2005). Many QTLs with minor additive effects are associated with a large difference in growth between two selection lines in chickens. Genet. Res. 86: 115-125.

Laclef C (2014). Primary cilia control different steps of brain development. Med. Sci. 30: 980-990.

Li R, Yu C, Li Y, Lam TW, et al. (2009). SOAP2: an improved ultrafast tool for short read alignment. Bioinformatics 25: 19661967.

Lindholm-Perry AK, Sexten AK, Kuehn LA, Smith TP, et al. (2011). Association, effects and validation of polymorphisms within the NCAPG-LCORL locus located on BAT6 with feed intake, gain, meat and carcass traits in beef cattle. BMC Genet. 12: 103.

Liu YZ, Wilson SG, Wang L, Liu XG, et al. (2008). Identification of PLCL1 gene for hip bone size variation in females in a genome-wide association study. PLoS One 3: e3160.

McCarthy MI, Abecasis GR, Cardon LR, Goldstein DB, et al. (2008). Genome-wide association studies for complex traits: consensus, uncertainty and challenges. Nat. Rev. Genet. 9: 356-369.

Moura A, Coutinho LL, Ambo M, Campos RLR, et al. (2009). Associations between microsatellite markers and traits related to performance, carcass and organs in chickens. Int. J. Poult. Sci. 8: 615-620.

Mulle C, Sailer A, Perez-Otano I, Dickinson-Anson H, et al. (1998). Altered synaptic physiology and reduced susceptibility to kainate-induced seizures in GluR6-deficient mice. Nature 392: 601-605.

Nicodemus KK, Liu W, Chase GA, Tsai YY, et al. (2005). Comparison of type I error for multiple test corrections in large single-nucleotide polymorphism studies using principal components versus haplotype blocking algorithms. BMC Genet. 6 (Suppl 1): S78.

Purcell S, Neale B, Todd-Brown K, Thomas L, et al. (2007). PLINK: A tool set for whole-genome association and populationbased linkage analyses. Am. J. Hum. Genet. 81: 559-575.

Ropka-Molik K, Zukowski K, Eckert R, Gurgul A, et al. (2014). Comprehensive analysis of the whole transcriptomes from two different pig breeds using RNA-seq method. Anim. Genet. 45: 674-684. 
Sewalem A, Morrice DM, Law A, Windsor D, et al. (2002). Mapping of quantitative trait loci for body weight at three, six, and nine weeks of age in a broiler layer cross. Poultry Sci. 81: 1775.

Sun X, Liu D, Zhang X, Li W, et al. (2013). SLAF-seq: an efficient method of large-scale De Novo SNP discovery and genotyping using high-throughput sequencing. PLoS One 8: e58700.

Teoh PL and Sharrocks AD (2014). WDR5, ASH2L, and RBBP5 control the efficiency of FOS transcript processing. Cell Mol. Biol. Lett. 19: 215-232.

Tsudzuki M, Onitsuka S, Akiyama R, Iwamizu M, et al. (2007). Identification of quantitative trait loci affecting shank length, body weight and carcass weight from the Japanese cockfighting chicken breed, Oh-Shamo (Japanese Large Game). Cytogenet. Genome Res. 117: 288-295.

Wahlberg P, Carlborg O, Foglio M, Tordoir X, et al. (2009). Genetic analysis of an F2 intercross between two chicken lines divergently selected for body-weight. BMC Genomics 10: 248.

Wang D, Sun Y, Stang P, Berlin JA, et al. (2009). Comparison of methods for correcting population stratification in a genomewide association study of rheumatoid arthritis: principal-component analysis versus multidimensional. BMC Proc. 3 (Suppl 7): S109.

Watson S-J, Brown AJ and Holliday ND (2012). Differential signaling by splice variants of the human free fatty acid receptor GPR120. Mol. Pharmacol. 81: 631-642.

Xie L, Luo CL, Zhang CG, Zhang R, et al. (2012). Genome-wide association study identified a narrow chromosome 1 region associated with chicken growth traits. PLoS One 7: e30910.

Xu X, Xu R and Zhu B (2015). A high-density genetic map of cucumber derived from Specific Length Amplified Fragment sequencing (SLAF-seq). Front. Plant Sci. 7: 768.

Zhang L, Zheng MQ, Liu RR, Wen J, et al. (2012). Genome-wide association of thymus and spleen mass in chicken. Sci. Agric. Sin. 45: 3165-3175. 